\title{
Ocorrência de retinopatia da prematuridade em recém-nascidos de muito baixo peso em maternidade de referência terciária no município de Fortaleza - CE
}

\section{Occurrence of retinopathy of prematurity in low-born newborn weights in tertiary reference maternity in the municipality of Fortaleza - CE}

\author{
Maria do Socorro Landim Rodrigues Alves Xavier ${ }^{1}$. Tania Maria Sousa Araújo Santos ${ }^{1,2}$. \\ 1 Maternidade Escola Assis Chateaubriand (MEAC), Universidade Federal do Ceará (UFC), Fortaleza, Ceará, Brasil. 2 Hospital \\ Infantil Albert Sabin, Fortaleza, Ceará, Brasil.
}

\begin{abstract}
RESUMO
Objetivo: conhecer as taxas de retinopatia da prematuridade (ROP) em recém-nascidos $(\mathrm{RN}) \leq 1500 \mathrm{~g}$ e/ou $\leq$ a 32 semanas, no serviço de neonatologia de uma maternidade pública terciária do município de Fortaleza. Métodos: estudo transversal, retrospectivo de dados secundários, onde os dados foram coletados nos prontuários no período de 1 ano, através de questionário simples. Resultados: nasceram 434 RN's e destes foram excluídos óbitos, transferências, malformações e infecções congênitas. Foram incluídos na pesquisa 119 prontuários. Destes, 31 (26\%) pacientes apresentaram ROP, com predominância do estágio II (61,3\%), zona II $(93,5 \%)$, sem plus $(64,5 \%)$, dos quais $45,2 \%$ realizaram tratamento com laser. Houve predomínio em prematuro extremo $(61,3 \%)$, peso menor que $1000 \mathrm{~g}(71 \%)$, sexo feminino $(54,8 \%)$ e parto vaginal $(61,3 \%)$. A maioria dos $\mathrm{RN}$ 's necessitaram de manobra de reanimação (67,7\%). 96,8\% apresentaram síndrome do desconforto respiratório (SDR), 77,4\% utilizaram surfactante e tiveram tempo de oxigenioterapia maior que 30 dias. A persistência do canal arterial (PCA) foi diagnosticada em 58,1\%, sepse clínica em 93,5\% e anemia em 96,8\%. Conclusão: a maioria dos casos de ROP foram em RN's prematuros com evolução intimamente relacionada a comorbidades comuns no período neonatal.
\end{abstract}

Palavras-chave: Recém-nascido de muito baixo peso. Retinopatia da prematuridade. Fatores de risco.

\begin{abstract}
Objective: To know the rates of retinopathy of prematurity(ROP) in newborns (NB) $\leq 1500 \mathrm{~g}$ and/or $\leq$ to 32 weeks, in the neonatal service of a tertiary public maternity hospital in the city of Fortaleza. Methods: A cross-sectional, retrospective study of secondary data, where data were collected in medical records in a 1-year period, using a simple questionnaire. Results: 434 newborns were born and of these, deaths, transfers, malformations and congenital infections were excluded. 119 medical records were included in the study. Of these, $31(26 \%)$ patients presented ROP, with predominance of stage II (61.3\%), zone II (93.5\%), without plus (64.5\%), of which $45.2 \%$ underwent laser treatment. There was a predominance of extreme premature $(61.3 \%)$, weight less than $1000 \mathrm{~g}(71 \%)$, female sex $(54.8 \%)$ and vaginal delivery $(61.3 \%)$. The majority of NBs required a resuscitation maneuver $(67.7 \%)$. $96.8 \%$ presented respiratory distress syndrome (RDS), $77.4 \%$ used surfactant and had oxygen therapy time greater than 30 days. Persistence of ductus arteriosus (PCA) was diagnosed in $58.1 \%$, clinical sepsis in $93.5 \%$ and anemia in $96.8 \%$. Conclusion: the majority of cases of ROP were in premature newborns with an evolution closely related to common comorbidities in the neonatal period.
\end{abstract}

Keywords: Very low birth weight newborn. Retinopathy of prematurity. Risk factors.

Autor correspondente: Maria do Socorro Landim Rodrigues Alves Xavier, Rua Jornalista Cesar Magalhães, 500, Guararapes, Parquelândia, Fortaleza, Ceará. CEP: 60810-140. Telefone: +55 85 99113-9696. E-mail: bia.marcosneto@hotmail.com

Conflito de interesses: Não há qualquer conflito de interesses por parte de qualquer um dos autores.

Recebido em: 25 Set 2018; Revisado em: 11 Nov 2018; Aceito em: 14 Nov 2018. 


\section{INTRODUÇÃO}

A retinopatia da prematuridade (ROP) constitui um problema clínico e de saúde pública de extrema relevância. Configura-se dentre as causas importantes de cegueira infantil que leva a repercussões no desenvolvimento global do recém-nascido (RN) prematuro, nos quais, observa-se a interrupção do processo natural de vasculogênese da retina. ${ }^{1,2}$

Esta condição relatada por Terry em 1942, como fibroplasiaretrolental, cuja fisiopatologia associa-se a variações de hipóxia local e fenômenos vasculares sistêmicos, ganhou visibilidade nos países desenvolvidos. ${ }^{1,2}$ Os avanços tecnológicos e científicos, aplicados às práticas assistenciais, como o uso indiscriminado de oxigênio e seu controle refletiram-se nas taxas através das décadas, desde a primeira epidemia com 7.000 casos nos Estados Unidos, em 1953 e com redução de taxas de ROP em 1950 (50\%) e 1964 $(04 \%,)^{3}$

A qualidade de assistência neonatal, incluindo recursos humanos, tecnológicos e processos, contribuíram para redução da mortalidade neonatal, mas ocorreu a segunda epidemia entre os anos 60 e 70 e a terceira epidemia de ROP a partir dos anos 80 com estimativa anual de 300 novos casos de cegueira por ROP, incluindo os países em desenvolvimento, dada a sobrevida de recém-nascidos extremos e muito baixo peso. ${ }^{3-8}$

No Brasil, os dados mostram crescimento nas taxas de ROP, mas variam bastante com inferências anuais de até 16.000 recém-nascidos portadores de ROP e ganham importância os programas de acompanhamento preventivo e terapêutico embora sejam acessíveis somente em centros de referência. ${ }^{1,6,9-11}$

A patogênese da ROP depende do fator de crescimento do endotélio vascular(VEGF), regulado pelo oxigênio e fatores não regulados pelo oxigênio como o fator de crescimento insulina like (IGFI). ${ }^{8}$ Patz e cols., em estudos clínicos prospectivos e controlados, observaram a relação de causa e efeito entre o uso de oxigenioterapia em ventilação mecânica e o aparecimento da ROP, entretanto, o nível de segurança no uso da oxigenioterapia em prematuros ainda não foi definido, apesar de notório saber que o uso cauteloso reduz a incidência de ROP. ${ }^{12}$

Sua etiologia é multifatorial com destaque para peso ao nascimento, idade gestacional e uso de oxigenioterapia, ${ }^{7,13-15}$ mas outros fatores de risco foram identificados no estudo retrospectivo realizado na Universidade de São Paulo (USP) como: uso de fototerapia, múltiplas transfusões sanguíneas, presença de hemorragia intracraniana e asfixia. . $^{3,10,11,16,17}$

O diagnóstico de ROP baseia-se na classificação internacional da retinopatia da prematuridade (ICROP)/CRYO-ROP (The Multicenter Trial of Cryotherapy for Retinopathy of Prematurity Study) que um grupo de experts elaborou e publicou nas versões de 1984 e 1987, a qual inclui a definição e estabelece a gravidade conforme os critérios: estadiamento (estágios 1-5), localização na retina (zonas I-III), extensão em horas (1-12h) e atividade da doença com ou sem a presença de doença plus (dilatação arteriolar e tortuosidade venosa) conforme Quadro 1.

Quadro 1. Classificação Internacional da Retinopatia da Prematuridade (1984/1987/2005). ${ }^{8}$

\begin{tabular}{|l|l|}
\hline \multicolumn{2}{|l|}{ Classificação Internacional da Retinopatia da Prematuridade $(1984 / 1987 / 2005)^{8}$} \\
\hline Estágio 1-ROP I & $\begin{array}{l}\text { Isquemia com presença de linha branca e plana que separa a retina } \\
\text { vascular da avascular }\end{array}$ \\
\hline Estágio 2-ROP II & $\begin{array}{l}\text { Alargamento da linha e identificação de crista elevada na retina } \\
\text { periférica }\end{array}$ \\
\hline Estágio 3-ROP III & $\begin{array}{l}\text { Proliferação fibrovascular a partir da crista com subdivisões a partir de } \\
\text { 2005: } \\
\text { ROP III leve } \\
\text { ROP III moderada } \\
\text { ROP III grave }\end{array}$ \\
\hline Estágio 4-ROP 4 & $\begin{array}{l}\text { A proliferação pode provocar um descolamento de retina subtotal } \\
\text { ROP 4A: descolamento parcial e não afeta a mácula; } \\
\text { ROP 4B: descolamento parcial que atinge a mácula }\end{array}$ \\
\hline $\begin{array}{l}\text { Estágio 5-ROP 5 } \\
\text { Doença limiar - indicação de tratamento pelo risco } \\
\text { desfavorável em 50\% - definido pelo CRYO-ROP (1988) }\end{array}$ & $\begin{array}{l}\text { Retinopatia estágio 3, em zona I ou II, com, pelo menos, cinco horas de } \\
\text { plus (dilatação arteriolar e venodilatação) }\end{array}$ \\
\hline
\end{tabular}

Continua 
Conclusão.

Quadro 1. Classificação Internacional da Retinopatia da Prematuridade (1984/1987/2005). ${ }^{8}$

\begin{tabular}{|l|l|}
\hline $\begin{array}{l}\text { Doença pré-limiar tipo 1 - definido risco e indicação de } \\
\text { tratamento ET-ROP (2003) }\end{array}$ & $\begin{array}{l}\text { Qualquer ROP em zona I com plus - doença posterior agressiva } \\
\text { Estágio 3, zona I, sem plus } \\
\text { Estágio 2 ou 3 em zona II, com plus }\end{array}$ \\
\hline Doença pré-limiar tipo 2 - Definido pelo ET-ROP & $\begin{array}{l}\text { Estágio 1 ou 2, zona I, sem plus } \\
\text { Estágio 3, zona II, sem plus }\end{array}$ \\
\hline ROP agressiva posterior - introdução em 2005 & Forma grave de rápida progressão sem padrão evolutivo de estadiamento \\
\hline
\end{tabular}

No Brasil, recomenda-se o uso das Diretrizes para a Triagem de Detecção e Tratamento da ROP em todos os recém-nascidos prematuros com peso de nascimento $(\mathrm{PN}) \leq 1.500$ gramas e/ ou idade gestacional $(\mathrm{IG}) \leq 32$ semanas ao nascimento. A primeira avaliação com retinólogo deve ser realizada entre a $4^{\mathrm{a}}$ e a $6^{\mathrm{a}}$ semana de vida, usando oftalmoscópio binocular indireta (OBI) e dilatação pupilar. Outros neonatos expostos a riscos para desenvolver a doença, também tem indicação do exame oftalmológico. ${ }^{5,7,18}$ As avaliações subsequentes e o aprazamento dos exames dependem dos achados e estadiamento, e seguem o protocolo, a saber: seguimento semestral para retina vascular; quinzenal para retina imatura ou presença de ROP pré-limiar; retinopatia em regressão e retina imatura em Zona I: semanal; ROP pré-limiar do tipo 1 (Zona I, qualquer estadiamento com plus; Zona I, Estadiamento 3; Zona II, Estadiamento 2 ou 3 plus) exame em 3 a 7 dias e ROP limiar: tratamento em até 72 horas. ${ }^{5,18}$ As avaliações descontinuam quando o bebê apresenta retina totalmente vascularizada, atinge a idade gestacional corrigida acima de 45 semanas sem ROP pré-limiar ou apresenta regressão completa da lesão.,18

Os RN classificados com ROP pré-limiar do tipo I são elegíveis para tratamento com laser aplicado com OBI entre as $37^{\mathrm{a}}-38^{\mathrm{a}}$ semanas de idade corrigida ${ }^{1,19}$ para preservar a qualidade de visão no paciente acometido e seguem em acompanhamento evolutivo semanal pela possibilidade de regressão em 14 a 21 dias do tratamento. Em caso de falha e evolução da doença plus (10 a 15\% dos casos) uma nova intervenção a laser pode ser considerada. ${ }^{20,21}$

Dentre as novas terapias para melhorar os desfechos visuais, utiliza-se injeção intravítrea de anti-VEGF para o tratamento da ROP grave, desde 2009 , entre a $35^{\mathrm{a}}$ ou $36^{\mathrm{a}}$ semana de idade pós-natal. ${ }^{1,22}$

A ROP estadio 1 ou 2, regride na maioria dos pacientes, pois a vascularização da retina periférica pode ir se completando, deixando poucas alterações anatômicas e funcionais residuais que não necessitam de intervenção. É necessário apenas acompanhamento durante os primeiros anos de vida, para a prevenção de ambliopia e do estrabismo..$^{1,5}$

Dada sua importância clínica, reflexo da atenção neonatal e a lacuna de dados locais, justifica-se o estudo para comparar com os dados epidemiológicas no Brasil. Optou-se em estudo retrospectivo sobre os recém-nascidos pré-termos de uma maternidade de referência terciária no município de Fortaleza - CE, no período de janeiro a dezembro de 2016 com o intuito de verificar: 1. Ocorrência anual da retinopatia nos recém-nascidos pré-termos; 2. Verificar a associação de ROP com os fatores de risco conhecidos.

\section{METODOLOGIA}

Estudo transversal, retrospectivo de dados secundários em prontuários no período de 1 de janeiro a 31 de dezembro de 2016. A amostra constituiu-se de RN's com peso de nascimento $\leq 1500 \mathrm{~g}$ e/ou $\leq 32$ semanas de idade gestacional, nascidos na Maternidade Escola Assis Chateaubriand (MEAC). O estudo realizou-se na unidade de neonatologia, nos serviços de terapia intensiva neonatal (UTIN), unidade de cuidados intermediários convencional (UCINCO) e unidade canguru (UCINCA). Foram incluídos todos os RN's nascidos pré-termos elegíveis segundo o protocolo do serviço e as diretrizes brasileiras de ROP. Foram excluídos os neonatos com alterações cromossômicas ou estruturais, portadores de infecções congênitas, os transferidos e os óbitos. O diagnóstico foi realizado por oftalmologista habilitado, utilizando aparelho oftalmoscópico e a retinopatia da prematuridade classificada em graus de acordo com a classificação internacional. Os exames foram realizados na beira do leito seguindo protocolo universal, que consiste em avaliar todos os recém-nascidos com peso $\leq 1500 \mathrm{~g}$ ou com idade gestacional $\leq 32$ semanas, entre a $4^{\mathrm{a}}$ e $6^{\mathrm{a}}$ semana de nascimento.

Utilizou-se um instrumento de coleta de dados com as seguintes variáveis: Variáveis maternas: idade, tipo de parto, realização de pré-natal, antecedentes maternos, idade gestacional. A idade gestacional foi calculada a partir do primeiro dia da última menstruação (DUM) e, quando duvidosa, foi considerado uma ecografia obstétrica precoce. $\mathrm{Na}$ ausência dos dois métodos anteriores, a idade gestacional foi estimada pelo exame físico do recém-nascido. Variáveis neonatais: sexo, peso do nascimento e classificação do recém-nascido. $\mathrm{O}$ peso de nascimento foi considerado aquele aferido na sala de parto. Os recém-nascidos foram agrupados por peso em faixas $(<1000 \mathrm{~g}, 1000-1500 \mathrm{~g}, 1500-2500 \mathrm{~g}$, $>2500 \mathrm{~g}$ ), para efeito de comparação das taxas de retinopatia da prematuridade. Variáveis perinatais e do processo de assistência: necessidade de reanimação, corticoide antenatal, 
suporte ventilatório e patologias associadas. As variáveis do processo de atenção na unidade de terapia intensiva neonatal (UTIN) foram o uso de ventilação mecânica invasiva (VMI) e tempo de oxigenioterapia.

As variáveis do processo de atenção relacionadas com a morbidade foram: presença da síndrome do desconforto respiratório (SDR), persistência do canal arterial (PCA), sepse clínica precoce ou tardia, anemia e icterícia. A SDR caracterizou-se clinicamente pela presença de desconforto respiratório de início logo após o nascimento ou nas primeiras horas de vida e piora progressiva nas primeiras 48 a 72 horas. O diagnóstico clínico foi confirmado por exames radiográficos que mostravam desde o padrão reticulogranular difuso à opacidade total dos campos pulmonares.

A PCA foi estabelecida por ecocardiograma transtorácico. A sepse neonatal foi estabelecida pela presença variável de sinais clínicos como distermias, letargia, apneia, sucção débil e irregular, associados a alterações da contagem leucocitária e/ ou plaquetas, aumento de neutrófilos imaturos em relação aos totais que resultem em um escore hematológico para triagem de sepse igual ou superior a três ou choque séptico. A presença de hemocultura positiva constituiu-se em alguns casos como confirmação diagnóstica.

Os dados foram consolidados utilizando-se o programa estatístico Epi Info, com o banco de dados constituído e apresentados em forma de frequência simples e tabelas.

Foram respeitados os princípios bioéticos com o objetivo de resguardar os direitos dos pacientes envolvidos no trabalho, de acordo com as questões expressas na Resolução 466/12 do Conselho Nacional de Saúde do Ministério da Saúde. O presente estudo foi avaliado pelo Comitê de Ética em Pesquisa envolvendo seres humanos da Maternidade Escola da Universidade Federal do Ceará.

\section{RESULTADOS}

No período de 01 de janeiro a 31 de dezembro de 2016 nasceram $434 \mathrm{RN}$ 's $\leq 32$ semanas e ou $\leq 1500$ g. Destes, 305 RN's foram excluídos por óbito, malformações, infecções congênitas e transferência. Foram revisados 129 prontuários dos pacientes submetidos ao exame oftalmológico, sendo 7 excluídos por óbito durante o período de avaliação e 3 por transferência durante o período de seguimento. Destes, foram incluídos na pesquisa 119 prontuários.

Dos 119 pacientes estudados, 31 apresentaram exame oftalmológico alterado (26\%). Da amostra incluída, houve predominância da ROP estágio $2(61,3 \%)$, zona II $(93,5 \%)$ e sem plus (64,5\%) (Tabela 1-2-3). Destes, 17 (54,8\%) não realizaram tratamento, apresentando vascularização com o seguimento e $14(45,2 \%)$ necessitaram de tratamento com laser, onde todos apresentaram resposta satisfatória após o tratamento.

Para permitir a análise quanto ao peso de nascimento e idade gestacional, na Tabela 4 estão distribuídas as prevalências da
ROP de acordo com as faixas de peso e de idade gestacional. Observa-se o maior contingente de RN's com ROP na faixa de peso menor que $1000 \mathrm{~g}(71 \%)$. Quanto à idade gestacional, constata-se uma elevação no grupo de 26 a 29 semanas $(61,3 \%)$ (Tabela 4). Houve predomínio do sexo feminino $(54,8 \%)$ e do parto vaginal $(61,3 \%)$.

Tabela 1. Frequência de ROP de acordo com a classificação internacional (ICROP).

\begin{tabular}{ll}
\hline Estadiamento & ROP $(\%)$ \\
\hline Estagio I & $16,1 \%$ \\
Estagio II & $61,3 \%$ \\
Estagio III & $22,6 \%$ \\
Estagio IV & $0 \%$ \\
Estagio V & $0 \%$ \\
\hline
\end{tabular}

Tabela 2. Frequência de ROP de acordo com a classificação internacional (ICROP).

\begin{tabular}{ll}
\hline Localização & ROP (\%) \\
\hline Zona I & $0 \%$ \\
Zona II & $93,5 \%$ \\
Zona III & $6,5 \%$ \\
\hline
\end{tabular}

Tabela 3. Frequência de ROP de acordo com a classificação internacional (ICROP).

\begin{tabular}{ll}
\hline Atividade da doença & ROP $(\%)$ \\
\hline Com plus & $35,5 \%$ \\
Sem plus & $64,5 \%$ \\
\hline
\end{tabular}

Tabela 4. Distribuição de ROP por faixas de peso e idade gestacional.

\begin{tabular}{|c|c|}
\hline Variáveis & ROP (n) (\%) \\
\hline \multicolumn{2}{|l|}{ Peso } \\
\hline$<1000$ gramas & $22(71 \%)$ \\
\hline 1000 - 1499 gramas & $6(19,4 \%)$ \\
\hline $1500-2500$ gramas & $3(9,7 \%)$ \\
\hline$>2500 \mathrm{~g}$ & 0 \\
\hline \multicolumn{2}{|l|}{ Idade gestacional } \\
\hline 23 - 25 semanas & $8(25,8 \%)$ \\
\hline $26-29$ semanas & $19(61,3 \%)$ \\
\hline $30-32$ semanas & $4(12,9 \%)$ \\
\hline$>32$ semanas & 0 \\
\hline
\end{tabular}


Com relação ao atendimento na sala de parto, verificou-se que a maioria dos RN's com ROP necessitaram de alguma manobra de reanimação $(67,7 \%)$, principalmente intubação orotraqueal. Nas variáveis do processo de atenção na UTIN, observou-se que $30(96,8 \%)$ pacientes apresentaram SDR, $24(77,4 \%)$ utilizaram surfactante e $24(77,4 \%)$ pacientes tiveram tempo de oxigenioterapia maior que 30 dias (Tabela 5-6). Todos os RN's realizaram ecocardiograma transtorácico e $18(58,1 \%)$ pacientes foram diagnosticados com PCA. A sepse clínica foi diagnosticada em 29 pacientes (93,5\%). Com relação à anemia, 30 pacientes $(96,8 \%)$ necessitaram de concentrado de hemácias, 29 (93,5\%) apresentaram ictérica com nível para fototerapia e $21(67,7 \%)$ apresentaram hemorragia perintraventricular (HPIV), principalmente grau I.

Tabela 5. Comorbidades associadas aos pacientes com ROP.

\begin{tabular}{ll}
\hline Comorbidades & ROP (\%) \\
\hline Manobras de reanimação $(\mathrm{n}=21)$ & $67,7 \%$ \\
SDR $(\mathrm{n}=30)$ & $96,8 \%$ \\
Surfactante $(\mathrm{n}=24)$ & $77,4 \%$ \\
PCA $(\mathrm{n}=29)$ & $93,5 \%$ \\
Sepse $(\mathrm{n}=29)$ & $93,5 \%$ \\
Anemia - transfusão $(\mathrm{n}=30)$ & $96,8 \%$ \\
Icterícia $(\mathrm{n}=29)$ & $93,5 \%$ \\
$* 2$ pacientes exsanguineotransfusão & \\
HPIV $(\mathrm{n}=21)$ & $67,7 \%$ \\
\hline
\end{tabular}

Tabela 6. Tempo de oxigenioterapia.

\begin{tabular}{ll}
\hline Tempo de oxigenioterapia & ROP $(\mathbf{n})(\%)$ \\
\hline$<15$ dias & $4(12,9 \%)$ \\
$15-30$ dias & $3(9,7 \%)$ \\
$>30$ dias & $24(77,4 \%)$ \\
\hline
\end{tabular}

\section{DISCUSSÃO}

Este estudo analisou RN's de muito baixo peso ao nascimento (RNMBPN) e menor ou igual a 32 semanas de idade gestacional nascidos na Maternidade Escola Assis Chateaubriand, no município de Fortaleza, Ceará. Dos 119 prontuários revisados, todos realizaram exame oftalmológico e 31 pacientes apresentaram retinopatia da prematuridade. Este contingente representa $26 \%$ do total de prontuários revisados, correspondendo à amostra final analisada. Desta amostra houve predominância da ROP estágio II (61,3\%), zona II $(93,5 \%)$ e sem plus $(64,5 \%)$.

A ROP representa uma das causas mais importantes de cegueira prevenível na infância. Seu aumento relaciona-se com elevação nas taxas de sobrevida de bebês muito prematuros assistidos nas unidades de terapia intensiva neonatais. Os casos de
ROP no serviço mostraram-se equivalentes à literatura, cuja prevalência varia de $13,2 \%$ a $36,4 \%$. $^{1,2,18}$

Encontrou-se expressiva associação das variáveis: peso ao nascimento, transfusão sanguínea, sepse, PCA e icterícia com o desenvolvimento de ROP em consonância com a literatura. ${ }^{4,710}$

No grupo com ROP, a média de peso foi de $938 \mathrm{~g}$, e a incidência foi maior no grupo de RN's com peso de nascimento menor que $1000 \mathrm{~g}(71 \%)$, frequência esta superestimada, mas que se justifica pela característica da amostra predominante de RN's de extremo baixo peso ao nascimento $(<1000 \mathrm{~g})$. Os dados demonstraram que quanto menor o peso, maior a chance em desenvolver a doença, o que está de acordo com a maioria dos estudos que apontam para o extremo baixo peso como fator de risco para aquisição da ROP.

O baixo peso como fator de risco sugere que fatores envolvidos no crescimento e desenvolvimento do indivíduo estejam implicados na etiologia dessa patologia. Trabalho feito em animais fundamentado em achados clínicos aponta o fator-1 de crescimento insulina-símile (IGF-1), o qual já é sabidamente importante no crescimento e desenvolvimento fetal, como participante fundamental na formação vascular retiniana. O nível sérico de IGF-1 se correlaciona com o crescimento fetal. Com isso, uma expressão menor desse fator contribuiria para um feto com peso reduzido e, portanto, com maior risco para ROP. ${ }^{19,23}$

No presente estudo, observou-se uma alta prevalência de SDR, uso de surfactante e uso de oxigenioterapia prolongada nos pacientes com ROP, que são compatíveis com outros estudos. Os níveis arteriais de oxigênio interferem na formação vascular retiniana do neonato. ${ }^{1,4,24}$

A vascularização normal da retina nasal se completa em média com 32 semanas, mas a vascularização da retina temporal se completa por volta de 40 semanas sob um regime de baixas pressões de oxigênio intraútero - $\mathrm{PaO}_{2}$ igual a $30 \mathrm{mmHg}$. Diante do parto prematuro e exposição a variadas frações de oxigênio eleva-se a pressão arterial de oxigênio $\left(\mathrm{PaO}_{2}\right)$ para 60 a $100 \mathrm{mmHg}$ e o RN pode desenvolver ROP, pois a hiperóxia pode interromper o processo normal, interferindo no fator de crescimento endotelial vascular (VEGF), inicialmente leva à hipóxia retiniana que estimula o aumento do VEGF e provoca uma neovascularização anômala. ${ }^{1,24}$

Vale destaque o fator IGF-I que exerce um papel necessário no processo de vascularização retiniana normal, não regulado pelo oxigênio, mas que em baixos níveis inibe a vascularização e se em excesso estimula a neovascularização. Segundo VillegasBecerril e cols. os níveis séricos de IGF-I podem ser utilizados como um fator para o rastreamento e monitoramento da ROP. ${ }^{25,26}$

Embora o uso de surfactante melhore outros desfechos, não se observou redução dos casos de ROP, provavelmente pela relação com imaturidade global e sua etiologia multifatorial. A duração da oxigenoterapia associou-se à maior ocorrência e gravidade da ROP. ${ }^{1,24,26}$ 
Observou-se que $58,1 \%$ dos pacientes com ROP apresentaram PCA e sepse neonatal (93,5\%). Fatores estes também apontados por Tartarella et al e relacionados ao desenvolvimento de ROP, seguido de anemia com necessidade de transfusão. ${ }^{1,15}$ A transfusão de concentrado de hemácias também expõe o prematuro ao desenvolvimento da doença, pelo desvio da curva de dissociação da hemoglobina e liberação de oxigênio aos tecidos, já constatado em outras publicações, além do risco de descompensação hemodinâmica. ${ }^{13}$ No presente estudo ainda ressaltou-se icterícia em 93,5\% dos casos em zona de fototerapia, sendo 2 casos com indicação de exsanguineotransfusão e 21 pacientes com $\operatorname{HPIV}(67,7 \%)$.

A identificação das características dos recém-nascidos prematuros, comorbidades e terapias utilizadas é fundamental para identificar o grupo de risco e implementar medidas efetivas na sua triagem, possibilitando a prevenção da cegueira e reduzindo a incidência da ROP ao longo do tempo.

\section{CONCLUSÃO}

Concluiu-se que a relação entre ROP e RNMBPN corresponde a $26 \%$ na população estudada, e todos estes casos foram diagnosticados com exame oftalmológico na beira do leito.

\section{REFERÊNCIAS}

1. Tartarella MB, Fortes JB Filho. Retinopatia da prematuridade. e-Oftalmo CBO: Rev Dig Oftalmol. 2016;2(4):1-16.

2. Carvalho DS, Moreira AT, Bonotto LB. Prevalência de retinopatia da prematuridade em prematuros atendidos no período de 1992-1999 em Joinville (SC): avaliação de riscos associados - "screening". Arq Bras Oftalmol. 2007;70(1):55-61.

3. Shinsato RN, Paccola L, Gonçalves WA, Barbosa JC, Martinez FE, Rodrigues ML, et al. Frequência de retinopatia da prematuridade em recém-nascidos no Hospital das Clínicas da Faculdade de Medicina de Ribeirão Preto da Universidade de São Paulo. Arq Bras Oftalmol. 2010;73(1):60-5.

4. Silva CF, Falco HC, Silva FG, Carvalho PK. Retinopatia da prematuridade: fatores de risco perinatais. Semina: Ciências Biológicas e da Saúde. 2016;37(1):3-14.

5. Associação Médica Brasileira; Conselho Federal de Medicina. Projeto diretrizes retinopatia da prematuridade [Internet]. São Paulo: Conselho Brasileiro de Oftalmologia e Sociedade Brasileira de Pediatria; 2011 [acesso em:20 Dez2015]. Disponível em: http://w.w.w.projetodiretrizes. org.br/diretrizes10/retinopatia_da_prematuridade.pdf

6. Theiss MB, Grumann JA, Rodrigues MR. Perfil epidemiológico dos recém-nascidos prematuros com retinopatia da prematuridade no Hospital Regional de São José Dr. Homero de Miranda Gomes. Rev Bras Oftalmol. 2016;75(2):109-14.

7. Fortes JB Filho, Eckert GU, Valiatti FB, Costa MC, Bonomo PP, Procianoy RS. Prevalência e fatores de risco para a retinopatia da prematuridade: estudo com 450 pré-termos de muito baixo peso. Rev Bras Oftalmol. 2009;68(1):22-9.

8. Fortes JB Filho. Medidas preventivas em retinopatia da
Ressalta-se que a maioria dos casos da ROP foram em recém-nascidos prematuros extremos $(61,3 \%)$, com evolução intimamente relacionada ao processo de atenção hospitalar (intubações, ventilação mecânica) e comorbidades no período neonatal (SDR, PCA, sepse, icterícia, anemia, HPIV), os quais elevam o nível de estresse do neonato, aumentam o tempo de oxigenioterapia e de período de internação, aumentando os riscos de desenvolver ROP.

A prevenção da prematuridade, otimização no tempo de oxigenioterapia e precisas indicações de transfusão sanguínea, principalmente, em unidades de terapia intensiva neonatais poderão contribuir para a redução futura da incidência de ROP. Sugerimos, a partir destes dados, elaborar um estudo de coorte prospectivo para acompanhamento dos fatores de risco associados a ROP nas unidades neonatais e assim promover melhor assistência ao RN.

O estudo realizado apresentou limitações quanto ao tamanho da amostra, que ao se apresentar em número reduzido devido ao elevado número de pacientes transferidos, permite considerar os resultados encontrados apenas para a população em questão.

prematuridade "seguimento ambulatorial do prematuro de risco". [place unknown]: Sociedade Brasileira de Pediatria; 2012 [acesso em: 12 Maio 2017]. Disponível em: http://www.sbop. com.br/webforms/Interna.aspx?secao_id=116\&s=Retinopatia-daPrematuridade-(ROP)\&c=Medidas-Preventivas-em-Retinopatia-daPrematuridade $\&$ campo $=295$

9. Brito PR, Veitzman S. Causas de cegueira e baixa visão em crianças. Arquivo Brasileiro Oftalmologia. 2000;63(1):49-54.

10. Englert JA, Saunders RA, Purohit D, Hulsey TC, Ebeling M. The effect of anemia on retinopathy of prematurity in extremely low birth weight infants. J Perinatol. 2001;21(1):21-6.

11. Aggarwal R, Agarwal R, Deorari AK, Paul VK. Retinopathy of prematurity. Indian J Pediatr. 2002;69(1):83-6.

12. Quinn GE. Retinopathy of prematurity in Brazil: an emerging problem. J Pediatr (Rio J). 2007;83(3):191-3.

13. Fortes JB Filho, Eckert GU, Procianoy L, Barros CK, Procianoy RS. Incidence and risk factors for retinopathy of prematurity in very low and in extremely low birth weight infants in a unit-based approach in southern Brazil. Eye (Lond). 2009;23(1):25-30.

14. Lad EM, Nguyen TC, Morton JM, Moshfeghi DM. Retinopathy of prematurity in the United States. Br J Ophthalmol. 2008;92(3):320-5.

15. Shah VA, Yeo CL, Ling YL, Ho LY. Incidence, risk factors of retinopathy of prematurity among very low birth weight infants in Singapore. Ann Acad Med Singapore. 2005;34(2):169-78.

16. Larsson E, Carle-Petrelius B, Cernerud G, Ots L, Wallin A, Holmström G. Incidence of ROP in two consecutive Swedish population based studies. Br J Ophthalmol. 2002;86(10):1122-6. Comment in: Br J Ophthalmol. 2002;86(10): 1071. 
17. Rodriguez-Hurtado FJ, Canizares JM. [Screening for retinopathy of prematurity. Our experience about limits of birth weight, postconceptional age and other risk factors]. Arch Soc Esp Oftalmol. 2006;81(5):275-9. Article in Spanish.

18. Sluncheva B, Vakrilova L, Emilova Z, Doicheva E, Topov A. [Retinopathy of prematurity - frequency and risk factors]. Akush Ginekol (Sofia). 2002;42(1):10-4. Article in Bulgarian.

19. Moraes NS, Graziano RM, Moreira CA Junior. Retinopatia da prematuridade. In: Abujamra S, Ávila M, Barsante C, Farah ME, Gonçalves JO, Lavinsky J, et al. Retina e vítreo: clínica e cirurgia. São Paulo: Roca; 2000. p.535-43.

20. Kim TI, Sohn J, Pi SY, Yoon YH. Postnatal risk factors of retinopathy of prematurity. Paediatr Perinat Epidemiol. 2004;18(2):130-4.

21. Nicoara SD, Nascutzy C, Cristian C, Irimescu I, Stefanut AC, Zaharie $\mathrm{G}$, et al. Outcomes and prognostic factors of intravitreal bevacizumab monotherapy in zone stage $3+$ and aggressive posterior retinopathy of prematurity. J Ophthalmol. 2015;2015:102582.
22. Hwang CK, Hubbard GB, Hutchinson AK, Lambert SR. Outcomes after intravitreal bevacizumab versus laser photocoagulation for retinopathy of prematurity: a 5-year retrospective analysis. Ophthalmology. 2015;122:1008.

23. Zin A, Florencio T, Fortes JB Filho, Nakanami CR, Gianini N, Graziano RM, et al. Brazilian guidelines proposal for screening and treatment of retinopathy of prematurity (ROP). Arq Bras Oftalmol. 2007;70(5):875-83.

24. Sears NC, Sears JE. Oxygen and retinopathy of prematurity. Int Ophthalmol Clin. 2011;51(1):17-31.

25. Villegas BE, Fernandez MF, Gonzalez R, Gallardo Galera JM. [Serum IGF-I levels in retinopathy of prematurity. New indications for ROP screening]. Arch Soc Esp Oftalmol. 2005;80(4):233-8. Article in Spanish.

26. Lermann VL, Fortes JB Filho, Procianoy RS. The prevalence of retinopathy of prematurity in very low birth weight newborn infants. J Pediatr (Rio J). 2006;82(1):27-32.

\section{Como citar:}

Xavier MS, Santos TM. Ocorrência de retinopatia da prematuridade em recém-nascidos de muito baixo peso em maternidade de referência terciária no município de Fortaleza - CE. Rev Med UFC. 2019 out-dez;59(4):7-13. 